\title{
Enhancement of ECG Signal
}

\author{
Hend Fat'hy \\ Modern academy for \\ Engineering and \\ Technology
}

\author{
Eman Mohamed, \\ $\mathrm{PhD}$ \\ Modern academy for \\ Engineering and \\ Technology
}

\author{
Ashraf Mohamed, \\ PhD \\ October University \\ forModern Sciences and \\ Arts
}

\author{
Wagdy Anis, PhD \\ Ain Shams University
}

\begin{abstract}
An Electrocardiogram (ECG) signal is a recording of the electrical activity of heart. It considered as an important source of vital diagnostic information. ECG signal is exposed to different types of noise. These noises change the nature of the ECG signal and provide difficulties on its analysis.
\end{abstract}

The one long Least Mean Squares (LMS) adaptive filter is an algorithm used to reduce the noise effect on the ECG signal. This algorithm is widely used in adaptive filter applications due to its simplicity and low computational complexity, but it suffers from low convergence speed.

This paper proposes to improve the one long LMS adaptive filter convergence speed using the multiple sub-adaptive filters proposed algorithm where simulations show that at MSE of 0.04 the required number of iterations are saved by about $4.3 * 10^{4}$ times compared to the one long LMS adaptive filter. Also comparison between them is performed in terms of Signal to Noise Ratio (SNR) against the step size $(\mu)$. It is found that the proposed algorithm provides improvement in the SNR by $5 \mathrm{~dB}$ at $\mu=0.2$.

The ECG samples are recorded from MIT-BIH database and an additive white Gaussian noise (AWGN) is added to the signal to examine the proposed technique and 2011a Mat lab platform is used to simulate these results.

\section{General terms}

ECG, Adaptive filter, one long LMS

\section{Keywords}

ECG, Adaptive filter, Noise reduction, one long LMS, multiple sub-filter, SNR and MSE

\section{INTRODUCTION}

The ECG signal is a diagnostic tool that graphically measures and records the electrical activity of heart in details[1]. ECG signal is used in the extraction of vital characteristics. ECG signal amplitude lies between 0.01 to $3 \mathrm{mv}$ and its frequency ranges from 0.5 to $100 \mathrm{~Hz}$ [2]. It consists of three pulses pwave, QRS complex and T-wave as represented in figure1.

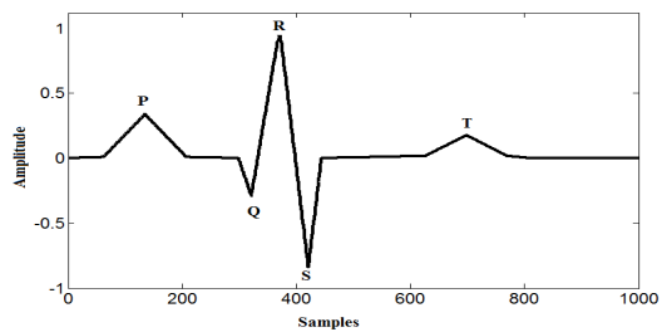

Figure 1 ECG signal

ECG signal suffer from different types of noise such as Power Line Interference (PLI), Base Line Wander (BW)andElectromyography (EMG). PLI generated when an
ECG machine is poorly grounded andits frequency value is 60 $\mathrm{HZ}$ and its multiples[3]. BW occurs due to Patient movement, dirty lead wires/electrodes, loose electrodes, respiration and perspiration. Its frequencyis lower than $0.5 \mathrm{HZ}$. EMG generated from muscles, it change from person to person and it is nearly has the same range of ECG signal frequency[4].It is important to produce ECG signal without noise in order to provide accurate diagnostic.

Adaptive filter techniques are suitable for filtering the EGG signal as it is random in nature[2].

\section{ADAPTIVE FILTER}

Adaptive filter algorithms are widely used for different applications such as noise cancellation, acoustic Echo cancellation, signal prediction and Channel equalization[5]. These filtersare self-designing digital filter as their coefficients are varying with time in order to minimize the error signal[6] .This error signal is the difference between desired signal and the filter output signal as;[5].

$e(n)=d(n)-y(n)$

Where, $\mathrm{X}(\mathrm{n})$ is the input signal , y (n) is the output signal, $\mathrm{d}(\mathrm{n})$ is the desired signal and $\mathrm{e}(\mathrm{n})$ is the error signal .This error signal used to control the adaptive filter algorithm in order to update the filter coefficients[7] as illustrated inFigure 2.

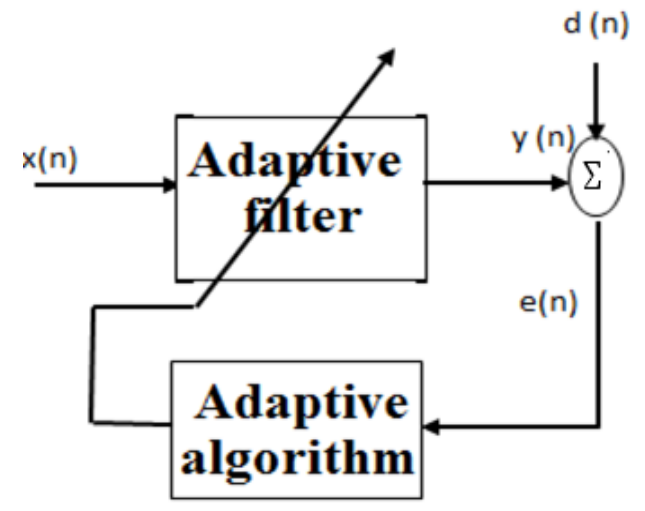

Figure 2 block diagram of adaptive filter.

Least Mean Square error (LMS), Normalized Least Mean Square error (NLMS), Recursive Least Mean Square error (RLS) and state-Space Recursive Least Mean Square error (SSRLS) are examples of adaptive filter algorithms[8]. These algorithms are different from each other by the relation which used to update the filter coefficients. The performance is evaluated according to:

- Convergence speed: This is determined by the number of iterations required for an algorithm to reach its desired MSE value[9]. 
- Misadjustment:The misadjustment is a dimensionless measure of the difference between the optimal performance and the actual performance of the LMS algorithm[10].

- Computational requirements: it is related to number of operations required to perform complete iteration of the algorithm and amount of memory needed to store the required data[11].

- Numerical Robustness: An adaptive filter algorithm is robust when its digital implementation using finite-word- length operation is stable[12].

The preferred adaptive filter algorithm should be robust, simple computations with higher convergence speed and small misadjustment. The stability and convergence speed are the most important parameters used to evaluate the adaptive algorithms[13].

\section{LMSADAPTIVE FILTER}

LMS is widely used adaptive filter due to its simplicity, low computational complexity and stability[14]. The coefficients of this filter are updated using the following formula:
$\mathrm{W}(\mathrm{n}+1)=\mathrm{w}(\mathrm{n})+\mu \mathrm{e}(\mathrm{n}) \mathrm{x}(\mathrm{n})$

Where $\quad w(n)=\left[w_{0}(n), w_{1}(n), \ldots, w_{P}(n)\right]^{T}$ the filter coefficients vector and $\mathrm{P}$ is is the filter order. This filter is updated at each iteration, and it is effected by pervious filter coefficients vector, step $\operatorname{size}(\mu)$ and calculated error signal [3].The range of step size is $0 \leq \mu<1 \quad$ LMS output vector is controlled using filter coefficients vector as following[15]:

$\mathrm{y}(\mathrm{n})=\mathrm{w}(\mathrm{n})^{\mathrm{T}} \mathrm{x}(\mathrm{n})$

LMS is a slow convergence speed filter, as it takes high time or needs large number of iterations in order to calculate the optimum filter coefficients that reduce MSE signal.

If fast convergence is desired, one should choose a large step size according to the stability limits[13].

The one long LMS adaptive filter is evaluated using 2011a Matlab platform. Figure 3 (a) represents the desired ECG signal for a normal patient. If EMG noise applied to this signal it will look like Figure 3 (b). EMG noise is equivalent to $10 \mathrm{~dB}$ AWGN. Figure 3 (c) illustrates the ECG signal after one long LMS filter with $\mu=0.2$ and $\mathrm{P}=10$.

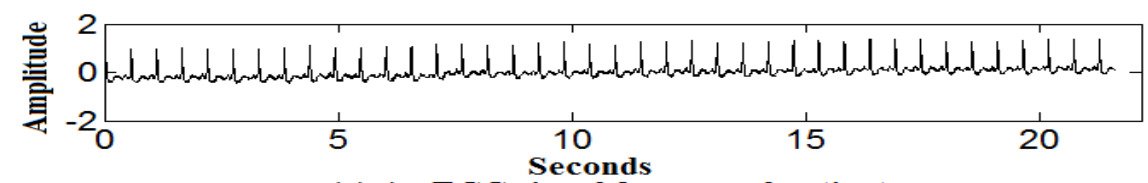

(a) An ECG signal for normal patient

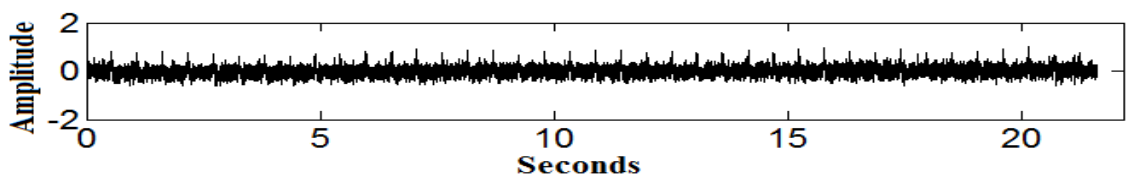

(b) An ECG signal for normal patient with AWGN $=10 d B$

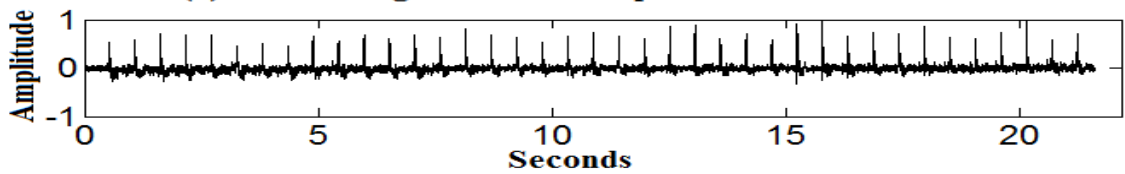

(C) The ECG signal after one long LMS adaptive filter

Figure 3the result of one long LMS for noisy ECG signal with input SNR 10dB

AWGN is the basic noise model[16]. AWGN is used as noise source for ECG signal to examine the one long LMS algorithm and the proposed technique.AWGN is generated by Matlab code with SNR $10 \mathrm{~dB}$. The noisy ECG signal is presented in figure 3 (b). This signal used as input to one long LMS adaptive filter algorithm and its SNR before filtering is equal to $-3.684 \mathrm{~dB}$. Figure 3 (c) represent the output of filter with SNR $=2.4302 \mathrm{~dB}$ so, noisy ECG signal is enhanced by $6.11 \mathrm{~dB}$.

\section{PROPOSED TECHNIQUE}

This paper proposed a multiple sub-adaptive filter to increase convergence speed of one long LMS adaptive filter[17]. This technique based on; decompose the one long LMS adaptive filter into three cascaded low order filters as shown in Figure 4. The filter order of three sub-filters is 2,6 and 2 respectively.

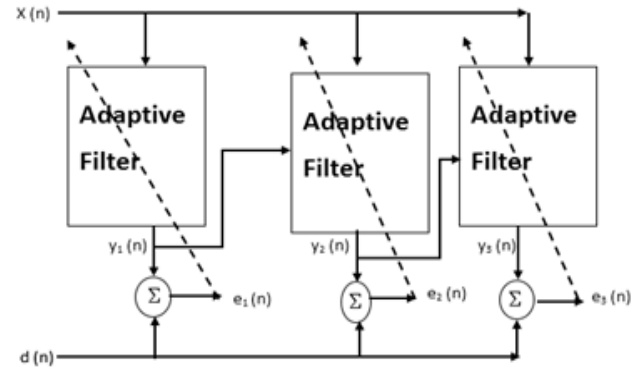

Figure 4the block diagram of the proposed technique

The operation of this filter based on, applying input signal to 1 st sub-filter. The output of this filter is filtered by 2 nd subfilter. Finally, the 2nd filtered signal applied to 3rdsub-filter in order to reduce its noise as:

$$
\begin{aligned}
& y_{1}(n)=w_{1}(n)^{T} x(n) \\
& e_{1}(n)=d(n)-y_{1}(n) \\
& w_{1}(n+1)=w_{1}(n)+\mu e_{1}(n) x(n)(6)
\end{aligned}
$$




$$
\begin{aligned}
& y_{2}(n)=w_{2}(n)^{T} y_{1}(n) \\
& e_{2}(n)=d(n)-y_{2}(n) \\
& w_{2}(n+1)=w_{2}(n)+\mu e_{2}(n) x(n) \\
& y_{3}(n)=w_{3}(n)^{T} y_{2}(n) \\
& e 3(n)=d(n)-y 3(n) \\
& \quad w_{3}(n+1)=w_{3}(n)+\mu e_{3}(n) x(n)(12
\end{aligned}
$$
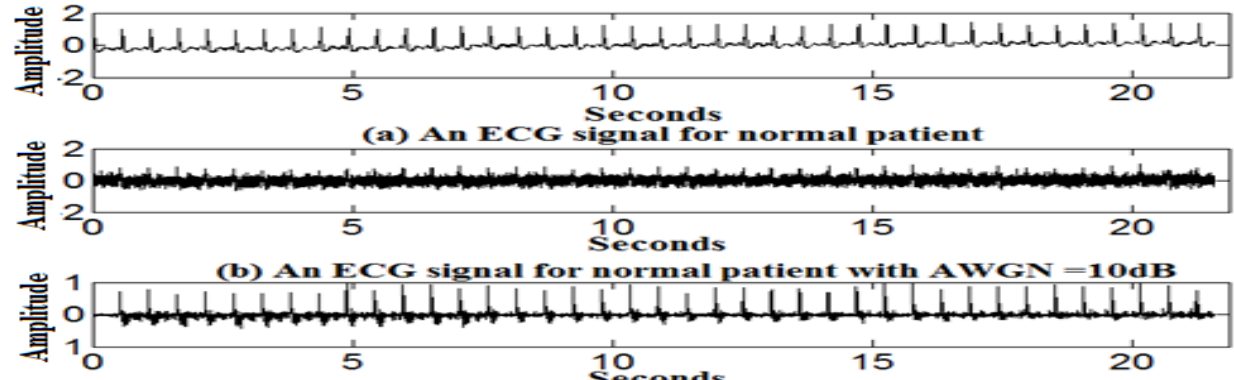

(c) The output of 1st sub-filer

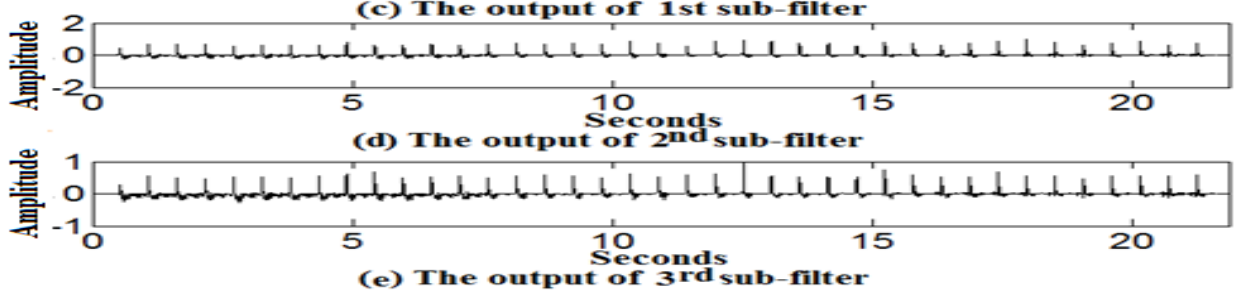

Figure 5 the results of proposed technique

Noisy ECG signal as in figure 5 (b) used as input to first 1st sub-filter algorithm with SNR before filtering $=-3.684 \mathrm{~dB}$. Figure 5 (c) represent the output of first 1st sub-filter with SNR $=4.1163 \mathrm{~dB}$ so, noisy ECG signal is enhanced by 7.8003 dB. Figure 5 (d)represents the output of second 2nd sub-filter with $\mathrm{SNR}=6.5478 \mathrm{~dB}$ so, noisy ECG signal is enhanced by $10.2318 \mathrm{~dB}$. Figure 5 (e)represent the output of third 3rd subfilter with $\mathrm{SNR}=7.4269 \mathrm{~dB}$ so, noisy $\mathrm{ECG}$ signal is enhanced by $11.1109 \mathrm{~dB}$.

From Figure 3 and Figure 5, the three multiple sub- adaptive LMS filter provides higher improvement in terms of SNR than one long LMS adaptive filter by $5 \mathrm{~dB}$.

\section{EVALUATION OF THE PROPOSED TECHNIQUE}

The one long LMS adaptive filter and proposed multiple subadaptive filter are evaluated and examine using the data base of ECG signal is used from MIT-BIH data base of patient (100) from physio bank ATM[18]. The sampling frequency of the recorded samples is $1000 \mathrm{~Hz}$. AWGN of $10 \mathrm{~dB}$ is added to the signal to examine the one long LMS adaptive filter and proposed technique. The tested signal consists of 22000 samples.

This paper aimed to design an adaptive filter with fast convergence speed and provide higher SNR. The convergence speed depends on the length of filter and step size $\mu[19]$. In this paper the filter length is fixed and different values of $\mu$ is used.

To evaluate performance of the proposed technique comparison between one long LMS adaptive filter and proposed technique is applied in terms of different values of $\mu$ and SNR with fixed filter length $\mathrm{p}=10$ for both one long LMS and proposed technique as illustrated in figure(6).

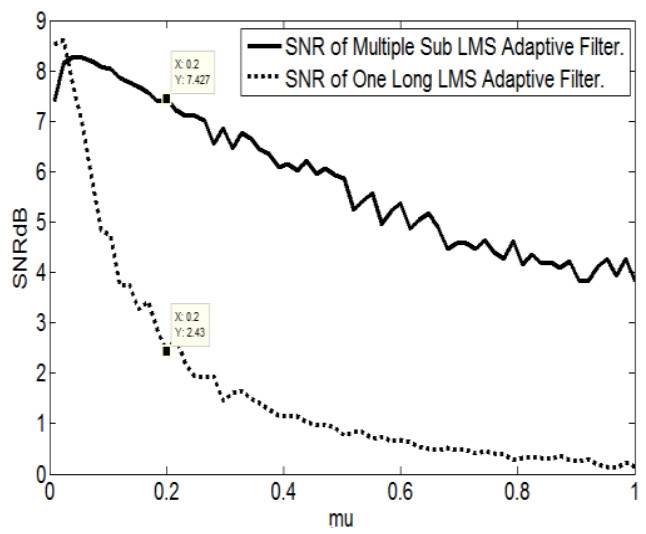

Figure 6a comparison between proposed technique and One Long LMS adaptive filters in terms of SNR for different mu

As shown in figure 6 it is Cleary that the proposed technique has SNR higher than the one long LMS algorithm for different values of step size $\mu$.Also the ECG signal for a sick Patient is used to evaluate the difference between one long LMS adaptive filter and the proposed technique.

Figure 7 a represents the desired ECG signal for a Sick patient. This signal applied to AWGN of $10 \mathrm{~dB}$ SNR so, its calculated SNR is $-3.4414 \mathrm{~dB}$ as presented in Figure 7 (b). Figure 7 (c) illustrates the output of one long LMS filter with $\mu=0.2$ and filter order $\mathrm{P}=10$. The calculated SNR of this filtered signal is $4.6781 \mathrm{~dB}$. This means that one long LMS adaptive filter enhance SNR of ECG signal by $8.1195 \mathrm{~dB}$. 


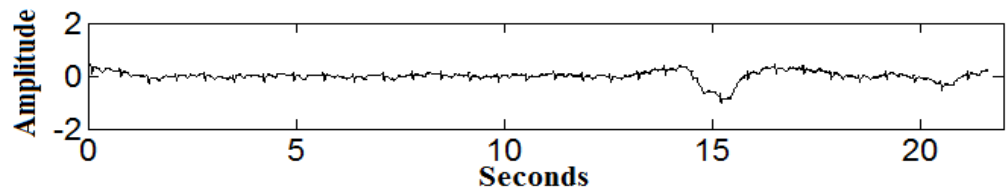

(a) An ECG signal for sick patient

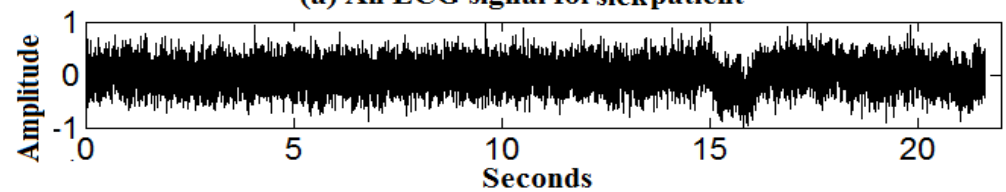

(b) An ECG signal for sick patient with AWGN =10dB

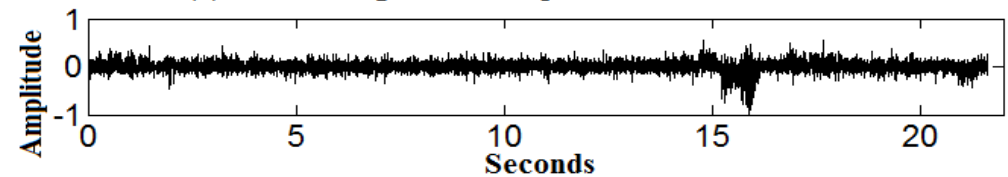

(C) The ECG signal after one long LMS adaptive filter

Figure 7 the result of one long LMS for noisy ECG signal with input SNR 10dB

Also this desired ECG signal and Noisy signal are applied to the proposed technique. Figure 8 (a) illustrates the output of first sub-filter with $\mu=0.2$ and filter order $p=2$ with $\mathrm{SNR}=3.8518 \mathrm{~dB}$. The first sub-filter enhances ECG by 7.2932 dB. Figure 8 (b) illustrates the output of second sub- filter with filter order $\mathrm{p}=6$ with $\mathrm{SNR}=3.7990$.the second sub-filter enhance ECG by $7.2403 \mathrm{~dB}$. Figure 8 (c) illustrates the output of third sub-filter withSNR=5.8851 dB. The third sub-filter enhances the ECGsignal by $9.3265 \mathrm{~dB}$.

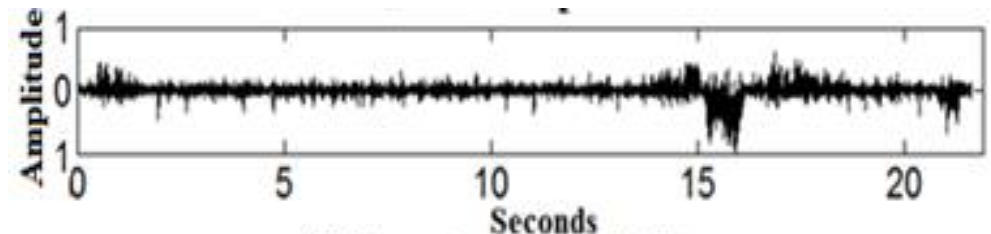

(a) The output of 1st sub-filter

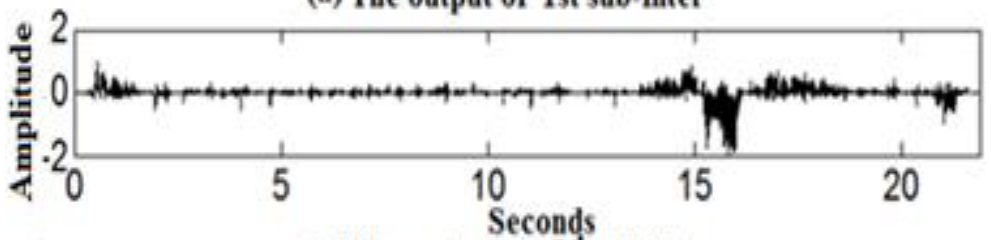

(b) The output of $2^{\text {nd }}$ sub-filter

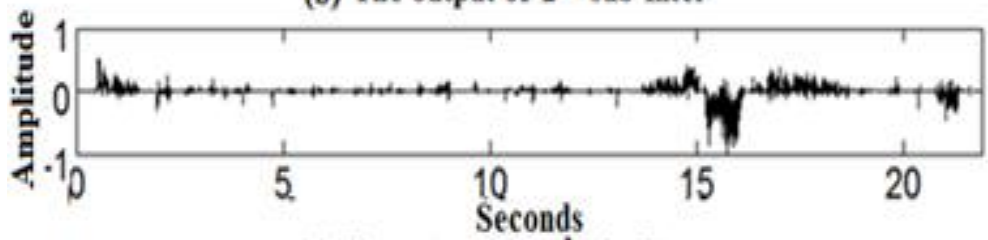

(c) The output of 3 rdsub-filter

Figure 8 the output of the proposed technique

From figure 7 and figure 8 , the three multiple sub-adaptive LMS filter provide higher improvement in terms of SNR than one long LMS adaptive filter by $1.207 \mathrm{~dB}$.
For normal patient and sick patient, the proposed technique has higher improvement in SNR than one long LMS adaptive filter as illustrates in table 1 and table 2 respectively.

Table 1 record of SNR for normal patient

\begin{tabular}{|c|c|c|c|c|c|c|c|}
\hline \multirow[b]{2}{*}{$\begin{array}{l}\text { Step } \\
\operatorname{size}(\mu)\end{array}$} & \multicolumn{5}{|c|}{ SNR } & \multicolumn{2}{|c|}{$\begin{array}{c}\text { Improvement in } \\
\text { SNR }\end{array}$} \\
\hline & $\begin{array}{c}\text { SNR } \\
\text { before } \\
\text { filter }\end{array}$ & $\begin{array}{c}\text { SNR } \\
\text { for one } \\
\text { long } \\
\text { LMS }\end{array}$ & $\begin{array}{c}\text { SNR } \\
\text { of } 1 \mathrm{st} \\
\text { sub- } \\
\text { filter }\end{array}$ & $\begin{array}{l}\text { SNR } \\
\text { of } 2 \text { nd sub- } \\
\text { filter }\end{array}$ & $\begin{array}{c}\text { SNR } \\
\text { of 3rd } \\
\text { sub- } \\
\text { filter }\end{array}$ & $\begin{array}{l}\text { One } \\
\text { long } \\
\text { LMS }\end{array}$ & $\begin{array}{l}3 \text { sub - } \\
\text { LMS }\end{array}$ \\
\hline 0.096 & -3.684 & 4.7932 & 4.7005 & 7.5825 & 8.0291 & 8.48 & 11.71 \\
\hline 0.2 & -3.684 & 2.4302 & 4.1163 & 6.5478 & 7.4269 & 6.11 & 11.11 \\
\hline
\end{tabular}


Table 2 record of SNR for sick patient

\begin{tabular}{|c|c|c|c|c|c|c|c|}
\hline Step size $(\mu)$ & \multicolumn{4}{|c|}{ SNR } & \multicolumn{2}{|c|}{$\begin{array}{c}\text { SNR } \\
\text { improvement }\end{array}$} \\
\hline & $\begin{array}{c}\text { SNR } \\
\text { before } \\
\text { filter }\end{array}$ & $\begin{array}{c}\text { SNR } \\
\text { for one long } \\
\text { LMS }\end{array}$ & $\begin{array}{c}\text { SNR } \\
\text { of 1st sub- } \\
\text { filter }\end{array}$ & $\begin{array}{c}\text { SNR } \\
\text { of 2nd sub- } \\
\text { filter }\end{array}$ & $\begin{array}{c}\text { SNR } \\
\text { of 3rd sub- } \\
\text { filter }\end{array}$ & $\begin{array}{c}\text { One } \\
\text { long } \\
\text { LMS }\end{array}$ & $\begin{array}{c}3 \\
\text { sub- } \\
\text { LMS }\end{array}$ \\
\hline 0.096 & -3.4414 & 6.0676 & 4.2750 & 5.3101 & 7.3267 & 9.51 & 10.8 \\
\hline 0.2 & -3.4414 & 4.6781 & 3.8518 & 3.7990 & 5.8851 & 8.12 & 9.33 \\
\hline
\end{tabular}

To examine the convergence speed number of iterations with MSE is plotted to compare between one long LMS adaptive filter and proposed technique. Figure 9 illustrates that comparison at certain value of $\mu=0.2$. At $\mathrm{MSE}=0.04$ the number of iteration required by proposed technique is $1.54 * 104$ but number of iteration required by one long LMS adaptive filter is $4.68 * 104$. The proposed technique is converge to its MSE faster thanone long LMS filter by $3.14 * 104$ times.

It is obvious that the proposed technique is higher than one long LMS adaptive filter in terms of MSE for largest number of iterations.

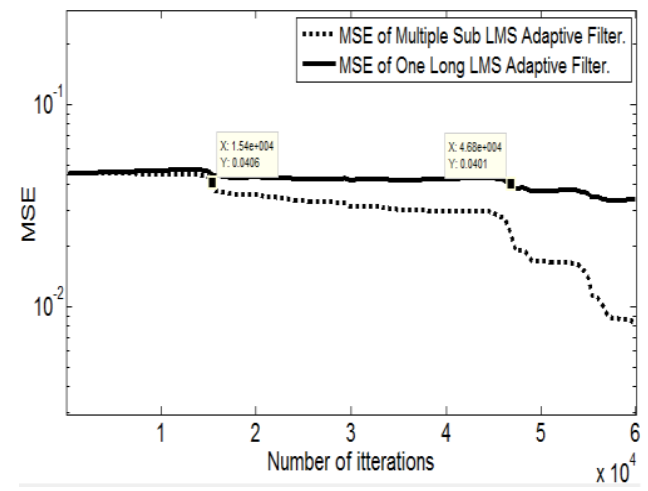

Figure 9a comparison between proposed technique and

One Long LMS adaptive filters in terms of MSE for different number of iterations

\section{CONCLUSION}

This Paper proposes to filter ECG signal to enhance it. Anew technique for multiple sub-filters is introduced. This proposed technique provides higher SNR than one long LMS adaptive filter. Also, it provides higher convergence speed. Using different types of adaptive filter algorithms will be suggested to increase the quality of ECG signal.

\section{REFERENCES}

[1] S.sharma, "noise reduction from ECG signal using adaptive filter algorithm," IJERT, vol. 3, no. 7, p. 6, 2014.

[2] V. R. Reddy, "Gaussian Noise Filtering From ECG Signal Using Improved Kalman Filter," International Journal of Engineering Research and Reviews, vol. 3, no. $2,2015$.

[3] M. Zia-Ur-Rahman, "Stationary and Non Stationary noise removal from Cardiac signals using aConstrained Stability Least Mean square Algorithm," IEEE, 2011.
[4] S. K. Alina Mirza, "Impulsive Noise Cancellation of ECG signal based on SSRLs," in Soft Computing and Software Engineering, 2015.

[5] P. S.R.Diniz, Adaptive Filtering Algorithms and Practicle Implimentation, New York: Springer Science +Business Media New York, 2013.

[6] S. Douglas, "Digital Signal proceeing," in Introduction to Adaptive Filters, 1999.

[7] M. d. C. a. P. J.A Apoliario Jr, "convergence analysis of the binormalized data-reusing LMS algprithm," IEEE Transaction on signal processing, November 2000.

[8] Sayrd.A.Hadei, "A family of Adaptive Filter Algorithms in Noise Cancellation for Speech Enhancement," IJCCEE, vol. 2, no. 2, April 2010.

[9] m. a. Ashraf, "Multiple sub-adaptive filter to acoustic echo cancellation and blind separation," 2009.

[10] j. s., "upper and lower of the misadjustment in the LMS algorithm," IEEE, 2002.

[11] Mr, "computational complexity of adaptive algoriyhm in echo cancellation," IJETE, vol. 2, no. 7, july 2015.

[12] seokchoi-Young, "robust regularization for Normalized LMS algorithm," IJETE, june 2014.

[13] A.H.B..M, "AdaVariable step size in LMS algorithm using Evolntionary programming," SPIJ, vol. 6, no. 2, 2012.

[14] B.boroujeny, Adaptive Filter Theory and Application, John Wiley \&sons, 2008.

[15] A.Zajnich, Principles of Adaptive filter and Self-learning Systems.

[16] A.I. M. R. a. A. T. I. Nuzhat Tanseem Awon, "Effect of AWGN \& Fading (Raleigh \& Rician) channels on BER performance of a WiMAX communication System," (IJCSIS) International Journal of Computer Science and Information Security, vol. 10, August 2012.

[17] S.Hussian, "Noise Removal from Cardiac Signals Using Various Adaptivealgoritms," in International Academic Conference on Electrical,Electronics and Computer Engineering, Chennai, 3,2013.

[18] "www.physio.org," [Online].

[19] "zone.ni.com," 1 2008. [Online]. 